\title{
LIMITS OF TANGENTS OF QUASI-ORDINARY HYPERSURFACES
}

\author{
ANTÓNIO ARAÚJO AND ORLANDO NETO
}

(Communicated by Lev Borisov)

\begin{abstract}
We compute explicitly the limits of tangents of a quasi-ordinary singularity in terms of its special monomials. We show that the set of limits of tangents of $Y$ is essentially a topological invariant of $Y$.
\end{abstract}

\section{INTRODUCTION}

The study of the limits of tangents of a complex hypersurface singularity was mainly developed by Lê Dũng Tráng and Bernard Teissier (see [4] and its bibliography). Chunsheng Ban [1] computed the set of limits of tangents $\Lambda$ of a quasiordinary singularity $Y$ when $Y$ has only one very special monomial (see Definition 1.2).

The main achievement of this paper is the explicit computation of the limits of tangents of an arbitrary quasi-ordinary hypersurface singularity (see Theorems 2.9, 2.10 and 2.11). Corollaries 2.12, 2.13 and 2.14 show that the set of limits of tangents of $Y$ comes quite close to being a topological invariant of $Y$. Corollary 2.13 shows that $\Lambda$ is a topological invariant of $Y$ when the tangent cone of $Y$ is a hyperplane. Corollary 2.15 shows that the triviality of the set of limits of tangents of $Y$ is a topological invariant of $Y$.

Let $X$ be a complex analytic manifold. Let $\pi: T^{*} X \rightarrow X$ be the cotangent bundle of $X$. Let $\Gamma$ be a germ of a Lagrangian variety of $T^{*} X$ at a point $\alpha$. We say that $\Gamma$ is in generic position if $\Gamma \cap \pi^{-1}(\pi(\alpha))=\mathbb{C} \alpha$. Let $Y$ be a hypersurface singularity of $X$. Let $\Gamma$ be the conormal $T_{Y}^{*} X$ of $Y$. The Lagrangian variety $\Gamma$ is in generic position if and only if $Y$ is the germ of a hypersurface with trivial set of limits of tangents.

Let $\mathcal{M}$ be a holonomic $\mathcal{D}_{X}$-module. The characteristic variety of $\mathcal{M}$ is a Lagrangian variety of $T^{*} X$. The characteristic varieties in generic position have a central role in $\mathcal{D}$-module theory (cf. Corollary 1.6.4 and Theorem 5.11 of $[6$ and Corollary 3.12 of [5]). It would be quite interesting to have good characterizations of the hypersurface singularities with trivial set of limits of tangents. Corollary 2.15 is a first step in this direction.

After finishing this paper, two questions arose naturally: Let $Y$ be a hypersurface singularity such that its tangent cone is a hyperplane. Is the set of limits of tangents of $Y$ a topological invariant of $Y$ ?

Received by the editors March 23, 2010 and, in revised form, March 7, 2011.

2010 Mathematics Subject Classification. Primary 14B05, 32S05.

This research was partially supported by FEDER and FCT-Plurianual 2010. 
Is the triviality of the set of limits of tangents of an hypersurface a topological invariant of the hypersurface?

Let $p: \mathbb{C}^{n+1} \rightarrow \mathbb{C}^{n}$ be the projection that takes $(x, y)=\left(x_{1}, \ldots, x_{n}, y\right)$ into $x$. Let $Y$ be the germ of a hypersurface of $\mathbb{C}^{n+1}$ defined by $f \in \mathbb{C}\left\{x_{1}, \ldots, x_{n}, y\right\}$. Let $W$ be the singular locus of $Y$. The set $Z$ defined by the equations $f=\partial f / \partial y=0$ is called the apparent contour of $f$ relative to the projection $p$. The set $\Delta=p(Z)$ is called the discriminant of $f$ relative to the projection $p$.

Near $q \in Y \backslash Z$ there is one and only one function $\varphi \in \mathcal{O}_{\mathbb{C}^{n+1}, q}$ such that $f(x, \varphi(x))=0$. The function $f$ defines implicitly $y$ as a function of $x$. Moreover,

$$
\frac{\partial y}{\partial x_{i}}=\frac{\partial \varphi}{\partial x_{i}}=-\frac{\partial f / \partial x_{i}}{\partial f / \partial y} \text { on } Y \backslash Z \text {. }
$$

Let $\theta=\xi_{1} d x_{1}+\cdots+\xi_{n} d x_{n}+\eta d y$ be the canonical 1-form of the cotangent bundle $T^{*} \mathbb{C}^{n+1}=\mathbb{C}^{n+1} \times \mathbb{C}_{n+1}$. An element of the projective cotangent bundle $\mathbb{P}^{*} \mathbb{C}^{n+1}=$ $\mathbb{C}^{n+1} \times \mathbb{P}_{n}$ is represented by the coordinates

$$
\left(x_{1}, \ldots, x_{n}, y ; \xi_{1}: \cdots: \xi_{n}: \eta\right) .
$$

We will consider in the open set $\{\eta \neq 0\}$ the chart

$$
\left(x_{1}, \ldots, x_{n}, y, p_{1}, \ldots, p_{n}\right),
$$

where $p_{i}=-\xi_{i} / \eta, 1 \leq i \leq n$. Let $\Gamma_{0}$ be the graph of the map from $Y \backslash W$ into $\mathbb{P}_{n}$ defined by

$$
(x, y) \mapsto\left(\frac{\partial f}{\partial x_{1}}: \cdots: \frac{\partial f}{\partial x_{n}}: \frac{\partial f}{\partial y}\right) .
$$

Let $\Gamma$ be the smallest closed analytic subset of $\mathbb{P}^{*} \mathbb{C}^{n+1}$ that contains $\Gamma_{0}$. The analytic set $\Gamma$ is a Legendrian subvariety of the contact manifold $\mathbb{P}^{*} \mathbb{C}^{n+1}$. The projective algebraic set $\Lambda=\Gamma \cap \pi^{-1}(0)$ is called the set of limits of tangents of $Y$.

Remark 1.1. It follows from (1.1) that

$$
\left(\frac{\partial f}{\partial x_{1}}: \cdots: \frac{\partial f}{\partial x_{n}}: \frac{\partial f}{\partial y}\right)=\left(-\frac{\partial y}{\partial x_{1}}: \cdots:-\frac{\partial y}{\partial x_{n}}: 1\right) \text { on } Y \backslash Z \text {. }
$$

Let $c_{1}, \ldots, c_{n}$ be positive integers. We will denote by $\mathbb{C}\left\{x_{1}^{1 / c_{1}}, \ldots, x_{n}^{1 / c_{n}}\right\}$ the $\mathbb{C}\left\{x_{1}, \ldots, x_{n}\right\}$ algebra given by the immersion from $\mathbb{C}\left\{x_{1}, \ldots, x_{n}\right\}$ into $\mathbb{C}\left\{t_{1}, \ldots, t_{n}\right\}$ that takes $x_{i}$ into $t_{i}^{c_{i}}, 1 \leq i \leq n$. We set $x_{i}^{1 / c_{i}}=t_{i}$. Let $a_{1}, \ldots, a_{n}$ be positive rationals. Set $a_{i}=b_{i} / c_{i}, 1 \leq i \leq n$, where $\left(b_{i}, c_{i}\right)=1$. Given a ramified monomial $M=x_{1}^{a_{1}} \cdots x_{n}^{a_{n}}=t_{1}^{b_{1}} \cdots t_{n}^{b_{n}}$ we set $\mathcal{O}(M)=\mathbb{C}\left\{x_{1}^{1 / c_{1}}, \ldots, x_{n}^{1 / c_{n}}\right\}$.

Let $Y$ be a germ at the origin of a complex hypersurface of $\mathbb{C}^{n+1}$. We say that $Y$ is a quasi-ordinary singularity if $\Delta$ is a divisor with normal crossings. We will assume that there is $l \leq m$ such that $\Delta=\left\{x_{1} \cdots x_{l}=0\right\}$.

If $Y$ is an irreducible quasi-ordinary singularity there are ramified monomials $N_{0}, N_{1}, \ldots, N_{m}, g_{i} \in \mathcal{O}\left(N_{i}\right), 0 \leq i \leq m$, such that $N_{0}=1, N_{i-1}$ divides $N_{i}$ in the ring $\mathcal{O}\left(N_{i}\right), g_{i}$ is a unit of $\mathcal{O}\left(N_{i}\right), 1 \leq i \leq m, g_{0}$ vanishes at the origin and the map $x \mapsto(x, \varphi(x))$ is a parametrization of $Y$ near the origin, where

$$
\varphi=g_{0}+N_{1} g_{1}+\cdots+N_{m} g_{m} .
$$

Replacing $y$ by $y-g_{0}$, we can assume that $g_{0}=0$. The monomials $N_{i}, 1 \leq i \leq m$, are unique and determine the topology of $Y$ (see [3]). They are called the special monomials of $f$. We set $\tilde{\mathcal{O}}=\mathcal{O}\left(N_{m}\right)$. 
Definition 1.2. We say that a special monomial $N_{i}, 1 \leq i \leq m$, is very special if $\left\{N_{i}=0\right\} \neq\left\{N_{i-1}=0\right\}$.

Let $M_{1}, \ldots, M_{g}$ be the very special monomials of $f$, where $M_{k}=N_{n_{k}}, 1=n_{1}<$ $n_{2}<\ldots<n_{g}, 1 \leq k \leq g$. Set $M_{0}=1, n_{g+1}=n_{g}+1$. There are units $f_{i}$ of $\mathcal{O}\left(N_{n_{i+1}-1}\right), 1 \leq i \leq g$, such that

$$
\begin{aligned}
& \varphi=M_{1} f_{1}+\cdots+M_{g} f_{g} . \\
& 2 . \text { LiMITS OF TANGENTS }
\end{aligned}
$$

\section{Limits OF TANGENTS}

After renaming the variables $x_{i}$ there are integers $m_{k}, 1 \leq k \leq g+1$, and positive rational numbers $a_{k i j}, 1 \leq k \leq g, 1 \leq i \leq k, 1 \leq j \leq m_{k}$ such that

$$
M_{k}=\prod_{i=1}^{k} \prod_{j=1}^{m_{k}} x_{i j}^{a_{k i j}}, \quad 1 \leq k \leq g .
$$

The canonical 1 -form of $\mathbb{P}^{*} \mathbb{C}^{n+1}$ becomes

$$
\theta=\sum_{i=1}^{g+1} \sum_{j=1}^{m_{i}} \xi_{i j} d x_{i j}
$$

We set $p_{i j}=-\xi_{i j} / \eta, 1 \leq i \leq g+1,1 \leq j \leq m_{i}$. Observe that

$$
\frac{\partial y}{\partial x_{i j}}=a_{i i j} \frac{M_{i}}{x_{i j}} \sigma_{i j}
$$

where $\sigma_{i j}$ is a unit of $\tilde{\mathcal{O}}$.

Theorem 2.1. If $\sum_{i=1}^{m_{1}} a_{11 i}<1$, then $\Lambda \subset\{\eta=0\}$.

Proof. Set $m=m_{1}, x_{i}=x_{1 i}, p_{i}=p_{1 i}, \xi_{i}=\xi_{1 i}$ and $a_{i}=a_{11 i}, 1 \leq i \leq m$. Given positive integers $c_{1}, \ldots, c_{m}$, it follows from (2.3) that

$$
\prod_{i=1}^{m} p_{i}^{c_{i}}=\prod_{i=1}^{m} x_{i}^{a_{i} \sum_{j=1}^{m} c_{j}-c_{i}} \phi,
$$

for some unit $\phi$ of $\tilde{\mathcal{O}}$. By (1.3) and (2.3),

$$
\phi(0)=f_{1}(0)^{\sum_{j=1}^{m} c_{j}} \prod_{j=1}^{m} a_{j}^{c_{j}} .
$$

Hence

$$
\eta^{\sum_{i=1}^{m} c_{i}}=\psi \prod_{i=1}^{m} \xi_{i}^{c_{i}} x_{i}^{c_{i}-a_{i} \sum_{j=1}^{m} c_{j}},
$$

for some unit $\psi$. If there are integers $c_{1}, \ldots, c_{m}$ such that the inequalities

$$
a_{k} \sum_{j=1}^{m} c_{j}<c_{k}, \quad 1 \leq k \leq m
$$

hold, then the result follows from (2.6). Hence it is enough to show that the set $\Omega$ of the $m$-tuples of rational numbers $\left(c_{1}, \ldots, c_{m}\right)$ that satisfy the inequalities (2.7) is nonempty. We will recursively define positive rational numbers $l_{j}, c_{j}, u_{j}$ such that

$$
l_{j}<c_{j}<u_{j},
$$


$j=1, \ldots, m$. Let $c_{1}, l_{1}, u_{1}$ be arbitrary positive rationals satisfying (2.8) 1 . Let $1<s \leq m$. If $l_{i}, c_{i}, u_{i}$ are defined for $i \leq s-1$, set

$$
l_{s}=\frac{a_{s} \sum_{j=1}^{s-1} c_{j}}{1-\sum_{j=s}^{m} a_{j}}, u_{s}=\left(a_{s} / a_{s-1}\right) c_{s-1} .
$$

Since $\sum_{j \geq s} a_{j}<1$ and

$$
\begin{aligned}
u_{s}-l_{s} & =\frac{a_{s}}{a_{s-1}\left(1-\sum_{j=s}^{m} a_{j}\right)}\left(\left(1-\sum_{j=s-1}^{m} a_{j}\right) c_{s-1}-a_{s-1} \sum_{j<s-1} c_{j}\right) \\
& =\frac{a_{s}}{a_{s-1}\left(1-\sum_{j=s}^{m} a_{j}\right)}\left(\left(1-\sum_{j=s-1}^{m} a_{j}\right)\left(c_{s-1}-l_{s-1}\right)\right),
\end{aligned}
$$

it follows from (2.8) $)_{s-1}$ that $l_{s}<u_{s}$. Let $c_{s}$ be a rational number such that $l_{s}<c_{s}<u_{s}$. Hence (2.8) $s$ holds for $s \leq m$.

Let us show that $\left(c_{1}, \ldots, c_{m}\right) \in \Omega$. Since $c_{k}<u_{k}$, then

$$
c_{k}<\frac{a_{k}}{a_{k-1}} c_{k-1} \text {, for } k \geq 2 \text {. }
$$

Then, for $j<k$,

$$
c_{k}<\frac{a_{k}}{a_{k-1}} \frac{a_{k-1}}{a_{k-2}} \cdots \frac{a_{j+1}}{a_{j}} c_{j}=\frac{a_{k}}{a_{j}} c_{j} .
$$

Hence,

$$
a_{k} c_{j}<a_{j} c_{k}, \text { for } j>k \text {. }
$$

Since $l_{k}<c_{k}$,

$$
a_{k} \sum_{j=1}^{k-1} c_{j}<c_{k}-\sum_{j=k}^{m} a_{j} c_{k}
$$

Hence, by (2.10),

$$
a_{k} \sum_{j=1}^{k-1} c_{j}<c_{k}-\sum_{j=k}^{m} a_{k} c_{j} .
$$

Therefore $a_{k} \sum_{j=1}^{m} c_{j}<c_{k}$.

Theorem 2.2. Let $1 \leq k \leq g$. Let $I \subset\left\{1, \ldots, m_{k}\right\}$. Assume that one of the following three hypotheses is satisfied:

(1) $\sum_{j \in I} a_{k k j}>1$;

(2) $k=1, \sum_{j \in I} a_{11 j}=1$ and $\sum_{j=1}^{m_{1}} a_{11 j}>1$;

(3) $k \geq 2$ and $\sum_{j \in I} a_{k k j}=1$.

Then $\Lambda \subset\left\{\prod_{j \in I} \xi_{k j}=0\right\}$.

Proof.

Case 1. We can assume that $I=\{1, \ldots, n\}$, where $1 \leq n \leq m_{k}$. Set $a_{i}=a_{k k i}$. Given positive integers $c_{1}, \ldots, c_{n}$, it follows from (2.3) that

$$
\prod_{i=1}^{n} \xi_{k i}^{c_{i}}=\prod_{i=1}^{n} x_{k i}^{a_{i} \sum_{j=1}^{n} c_{j}-c_{i}} \eta^{\sum_{i=1}^{n} c_{i}} \varepsilon
$$


where $\varepsilon \in \widetilde{\mathcal{O}}$. Hence it is enough to show that there are positive rational numbers $c_{1}, \ldots, c_{n}$ such that

$$
a_{k}\left(\sum_{j=1}^{n} c_{j}\right)-c_{k}>0,1 \leq k \leq n .
$$

We will recursively define $\left.\left.l_{j}, c_{j}, u_{j} \in\right] 0,+\infty\right]$ such that $c_{j}, l_{j} \in \mathbb{Q}$,

$$
l_{j}<c_{j}<u_{j}
$$

$j=1, \ldots, n$, and $u_{j} \in \mathbb{Q}$ if and only if $\sum_{i=j}^{n} a_{i}<1$. Choose $c_{1}, l_{1}, u_{1}$ satisfying (2.13). Let $1<s \leq n-1$. Suppose that $l_{i}, c_{i}, u_{i}$ are defined for $1 \leq i \leq s-1$. If $\sum_{j=s}^{n} a_{j}<1$, set

$$
l_{s}=\left(a_{s} / a_{s-1}\right) c_{s-1}, u_{s}=\frac{a_{s} \sum_{j=1}^{s-1} c_{j}}{1-\sum_{j=s}^{n} a_{j}} .
$$

Since

$$
\begin{aligned}
u_{s}-l_{s} & =\frac{a_{s}}{a_{s-1}\left(1-\sum_{j=s}^{n} a_{j}\right)}\left(a_{s-1} \sum_{j=1}^{s-2} c_{j}-c_{s-1}\left(1-\sum_{j=s-1}^{n} a_{j}\right)\right) \\
& \leq \frac{a_{s}}{a_{s-1}\left(1-\sum_{j=s}^{n} a_{j}\right)}\left(\left(1-\sum_{j=s-1}^{n} a_{j}\right)\left(u_{s-1}-c_{s-1}\right)\right),
\end{aligned}
$$

it follows from (2.13) $)_{s-1}$ that $l_{s}<u_{s}$.

If $\sum_{j=s}^{n} a_{j} \geq 1$, set $l_{s}$ as above and $u_{s}=+\infty$. We choose a rational number $c_{s}$ such that $l_{s}<c_{s}<u_{s}$. Hence (2.13) $)_{s}$ holds for $1 \leq s \leq n$.

Let us show that $c_{1}, \ldots, c_{n}$ satisfy (2.12). We will proceed by induction. First we will show that $c_{1}, \ldots, c_{n}$ satisfy $(2.12)_{n}$. Suppose that $a_{n}<1$. Since $c_{n}<u_{n}$, we have that

$$
c_{n}<\frac{a_{n} \sum_{j=1}^{n-1} c_{j}}{1-a_{n}} .
$$

Hence $a_{n} \sum_{j=1}^{n} c_{j}>c_{n}$. If $a_{n} \geq 1$, then

$$
a_{n} \sum_{j=1}^{n} c_{j} \geq \sum_{j=1}^{n} c_{j}>c_{n} .
$$

Hence (2.12) $n$ is satisfied. Assume that $c_{1}, \ldots, c_{n}$ satisfy $(2.12)_{k}, 2 \leq k \leq n$. Since $c_{k}>l_{k}$,

$$
a_{k} \sum_{j=1}^{n} c_{j}>c_{k}>\frac{a_{k}}{a_{k-1}} c_{k-1} .
$$

Hence $a_{k-1} \sum_{j=1}^{n} c_{j}>c_{k-1}$. Therefore $\left(c_{1}, \ldots, c_{n}\right)$ satisfy $(2.12){ }_{k-1}$.

Case 2. Set $a_{j}=a_{11 j}$ and $x_{j}=x_{1 j}$. We can assume that $I=\{1, \ldots, n\}$, where $1 \leq n \leq m_{1}$. Given positive integers $c_{1}, \ldots, c_{n}$, it follows from (1.2) that

$$
\prod_{i=1}^{n} \xi_{i}^{c_{i}}=\prod_{i=1}^{n} x_{i}^{a_{i} \sum_{j=1}^{n} c_{j}-c_{i}} \eta^{\sum_{i=1}^{n} c_{i}} \varepsilon
$$


where $\varepsilon \in \widetilde{\mathcal{O}}$ and $\varepsilon(0)=0$. Hence it is enough to show that there are positive rational numbers $c_{1}, \ldots, c_{n}$, such that

$$
a_{k} \sum_{j=1}^{n} c_{j}=c_{k}, 1 \leq k \leq n
$$

We choose an arbitrary positive integer $c_{1}$. Let $1<s \leq n$. If the $c_{i}$ are defined for $i<s$, set

$$
c_{s}=\frac{a_{s}}{a_{s-1}} c_{s-1}
$$

Let us show that $c_{1}, \ldots, c_{n}$ satisfy (2.16). We will proceed by induction in $k$. First let us show that $(2.16)_{n}$ holds.

Let $j<n-1$. By (2.17),

$$
c_{n-1}=\frac{a_{n-1}}{a_{n-2}} \frac{a_{n-2}}{a_{n-3}} \cdots \frac{a_{j+1}}{a_{j}} c_{j}=\frac{a_{n-1}}{a_{j}} c_{j} .
$$

By (2.17), and since $\sum_{j=1}^{n} a_{j}=1$,

$$
c_{n}=\frac{a_{n}}{a_{n-1}} c_{n-1}=\frac{c_{n-1}}{a_{n-1}}\left(1-\sum_{j=1}^{n-1} a_{j}\right)=\frac{c_{n-1}}{a_{n-1}}-\sum_{j=1}^{n-1} \frac{a_{j}}{a_{n-1}} c_{n-1} .
$$

Hence, by (2.18),

$$
c_{n}=\frac{c_{n-1}}{a_{n-1}}-\sum_{j=1}^{n-1} c_{j} .
$$

Therefore, $\sum_{j=1}^{n} c_{j}=c_{n-1} / a_{n-1}$. Hence by (2.17),

$$
a_{n} \sum_{j=1}^{n} c_{j}=a_{n} \frac{c_{n-1}}{a_{n-1}}=c_{n}
$$

Therefore (2.16) ${ }_{n}$ holds.

Assume (2.16) $k$ holds, for $2 \leq k \leq n$. Then

$$
a_{k} \sum_{j=1}^{n} c_{j}=c_{k}=\frac{a_{k}}{a_{k-1}} c_{k-1} .
$$

Hence, $a_{k-1} \sum_{j=1}^{n} c_{j}=c_{k-1}$.

Case 3 . We can assume that $I=\{1, \ldots, n\}$, where $1 \leq n \leq m_{k}$. Given positive integers $c_{1}, \ldots, c_{n}$, it follows from (2.3) that

$$
\prod_{i=1}^{n} \xi_{k i}^{c_{i}}=\left(\prod_{i=1}^{n} x_{k i}^{a_{k k i}\left(\sum_{j=1}^{n} c_{j}\right)-c_{i}}\right) \eta^{\sum_{i=1}^{n} c_{i}} \varepsilon
$$

where $\varepsilon \in \tilde{\mathcal{O}}$ and $\varepsilon(0)=0$. We have reduced the problem to case 2 .

Theorem 2.3. If $\sum_{k=1}^{m_{1}} a_{11 j}=1, \Lambda$ is contained in a cone. 
Proof. Set $a_{i}=a_{11 i}, i=1, \ldots, m_{1}$. Given positive integers $c_{1}, \ldots, c_{m_{1}}$, there is a unit $\phi$ of $\tilde{\mathcal{O}}$ such that

$$
\prod_{i=1}^{m_{1}} \xi_{i}^{c_{i}}=(-1)^{\sum_{j=1}^{m_{1}} c_{j}} \phi \prod_{i=1}^{m_{1}} x_{i}^{\sum_{j=1}^{m_{1}} c_{j} a_{i}-c_{i}} \eta^{\sum_{j=1}^{m_{1}} c_{j}} .
$$

By the proof of case 2 of Theorem 2.2. there is one and only one $m_{1}$-tuple of integers $c_{1}, \ldots, c_{m_{1}}$ such that $\left(c_{1}, \ldots, c_{m_{1}}\right)=(1), a_{i} \sum_{j=1}^{m_{1}} c_{j}=c_{i}, 1 \leq i \leq m_{1}$, and $\Lambda$ is contained in the cone defined by the equation

$$
\prod_{i=1}^{m_{1}} \xi_{i}^{c_{i}}-(-1)^{\sum_{j=1}^{m_{1}} c_{j}} \phi(0) \eta^{\sum_{j=1}^{m_{1}} c_{j}}=0,
$$

where $\phi(0)$ is given by (2.5).

Remark 2.4. Set $D_{\varepsilon}^{*}=\{x \in \mathbb{C}: 0<|x|<\varepsilon\}$, where $0<\varepsilon \ll 1$. Set $\mu=\sum_{k=1}^{g+1} m_{k}$. Let $\sigma: \mathbb{C} \rightarrow \mathbb{C}^{\mu}$ be a weighted homogeneous curve parametrized by

$$
\sigma(t)=\left(\varepsilon_{k i} t^{\alpha_{k i}}\right)_{1 \leq k \leq g+1,1 \leq i \leq m_{k}} .
$$

Notice that the image of $\sigma$ is contained in $\mathbb{C}^{\mu} \backslash \Delta$. Set $\theta_{0}(t)=1$ and

$$
\theta_{k i}(t)=\frac{\partial \varphi}{\partial x_{k i}}(\sigma(t), \varphi(\sigma(t))), 1 \leq k \leq g+1,1 \leq i \leq m_{k},
$$

for $t \in D_{\varepsilon}^{*}$. The curve $\sigma$ induces a map from $D_{\varepsilon}^{*}$ into $\Gamma$ defined by

$$
t \mapsto\left(\sigma(t), \varphi(\sigma(t)) ; \theta_{11}(t): \cdots: \theta_{g+1, m_{g}+1}(t): \theta_{0}(t)\right) .
$$

Let $\vartheta: D_{\varepsilon}^{*} \rightarrow \mathbb{P}^{\mu}$ be the map defined by

$$
t \mapsto\left(\theta_{11}(t): \cdots: \theta_{g+1, m_{g}+1}(t): \theta_{0}(t)\right) .
$$

The limit when $t \rightarrow 0$ of $\vartheta(t)$ belongs to $\Lambda$. The functions $\theta_{k i}$ are ramified Laurent series of finite type in the variable $t$. Let $h$ a be ramified Laurent series of finite type. If $h=0$, we set $v(h)=\infty$. If $h \neq 0$, we set $v(h)=\alpha$, where $\alpha$ is the only rational number such that $\lim _{t \rightarrow 0} t^{-\alpha} h(t) \in \mathbb{C} \backslash\{0\}$. We call $\alpha$ the valuation of $h$. Notice that the limit of $\vartheta$ depends only on the functions $\theta_{k i}, \theta_{0}$ of minimal valuation. Moreover, the limit of $\vartheta$ depends only on the coefficients of the term of minimal valuation of each $\theta_{i j}, \theta_{0}$. Hence the limit of $\vartheta$ depends only on the coefficients of the very special monomials of $f$. We can assume that $m_{g+1}=0$ and that there are $\lambda_{k} \in \mathbb{C} \backslash\{0\}, 1 \leq k \leq g$, such that

$$
\varphi=\sum_{k=1}^{g} \lambda_{k} M_{k}
$$

Remark 2.5. The set of limits of tangents of a plane curve is a finite set, the union of the tangent cones of its irreducible components. If the curve is irreducible, its limit of tangents is always trivial. The set of limits of tangents of a surface $Y$ is the union of the dual variety of the tangent cone of $Y$ with a finite family of projective lines. When we consider a hypersurface $Y$ of $\mathbb{C}^{n}$ with $n \geq 4$, the set of limits of tangents $\Lambda$ of $Y$ has a more complex structure. In order to describe $\Lambda$, Lê and Teissier introduced a finite family of subsets of the tangent cone of $Y$, the halo of $Y$. The halo of $Y$ is called "la auréole" of $Y$ in 4 . 
Remark 2.6. Let $L$ be a finite set. Set $\mathbb{C}^{L}=\left\{\left(x_{a}\right)_{a \in L}: x_{a} \in \mathbb{C}\right\}$. Let $\sum_{a \in L} \xi_{a} d x_{a}$ be the canonical 1-form of $T^{*} \mathbb{C}^{L}$. Let $\Lambda$ be the subset of $\mathbb{P}_{L}$ defined by the equations

$$
\prod_{a \in I} \xi_{a}=0, I \in \mathcal{I}
$$

where $\mathcal{I} \subset \mathcal{P}(L)$. Set $\mathcal{I}^{\prime}=\{J \subset L: J \cap I \neq \emptyset$ for all $I \in \mathcal{I}\}, \mathcal{I}^{*}=\left\{J \in \mathcal{I}^{\prime}\right.$ such that there is no $\left.K \in \mathcal{I}^{\prime}: K \subset J, K \neq J\right\}$. The irreducible components of $\Lambda$ are the linear projective sets $\Lambda_{J}, J \in \mathcal{I}^{*}$, where $\Lambda_{J}$ is defined by the equations

$$
\xi_{a}=0, \quad a \in J
$$

Let $Y$ be a germ of hypersurface of $\left(\mathbb{C}^{L}, 0\right)$. Let $\Lambda$ be the set of limits of tangents of $Y$. For each irreducible component $\Lambda_{J}$ of $\Lambda$ there is a cone $V_{J}$ contained in the tangent cone of $Y$ such that $\Lambda_{J}$ is the dual of the projectivization of $V_{J}$. The halo of $Y$ is the union of the cones $V_{J}, J \in \mathcal{I}^{*}$.

Remark 2.7. If $\Lambda$ is defined by the equations (2.23), the halo of $Y$ equals the union of the linear subsets $V_{J}, J \in \mathcal{I}^{*}$ of $\mathbb{C}^{L}$, where $V_{J}$ is defined by the equations

$$
x_{a}=0, \quad a \in L \backslash J .
$$

Lemma 2.8. The determinant of the $n \times n$ matrix $\left(\lambda_{i}-\delta_{i j}\right)$ equals

$$
(-1)^{n}\left(1-\sum_{i=1}^{n} \lambda_{i}\right)
$$

Proof. Notice that $\operatorname{det}\left(\lambda_{i}-\delta_{i j}\right)=$

$$
\left|\begin{array}{ccc|c} 
& & & 1 \\
& -I_{n-1} & & \vdots \\
& & & 1 \\
\hline \lambda_{1} & \cdots & \lambda_{n-1} & \lambda_{n}-1
\end{array}\right|=\left|\begin{array}{ccc|c} 
& & & 1 \\
& -I_{n-1} & & \vdots \\
& & \\
\hline 0 & \ldots & 0 & \sum_{i=1}^{n} \lambda_{i}-1
\end{array}\right| .
$$

Theorem 2.9. Assume that $\sum_{i=1}^{m_{1}} a_{11 i}<1$. Set

$$
L=\bigcup_{k=2}^{g}\{k\} \times\left\{1, \ldots, m_{k}\right\}, \mathcal{I}=\bigcup_{k=2}^{g}\left\{\{k\} \times I: \sum_{j \in I} a_{k k j} \geq 1\right\} .
$$

The set $\Lambda$ is the union of the irreducible linear projective sets $\Lambda_{J}, J \in \mathcal{I}^{*}$, defined by the equations $\eta=0$ and

$$
\xi_{k j}=0,(k, j) \in J .
$$

The tangent cone of $Y$ equals $\left\{x_{11} \cdots x_{1 m_{1}}=0\right\}$. The halo of $Y$ is the union of the cones $V_{J}, J \in \mathcal{I}^{*}$, where $V_{J}$ is defined by the equations $x_{1 j}=0,1 \leq j \leq m_{1}$, and

$$
x_{k j}=0,(k, j) \in L \backslash J .
$$

Proof. Let us show that $\Lambda_{J} \subset \Lambda$. We can assume that there are integers $n_{1}, \ldots, n_{g}$, $1 \leq n_{k} \leq m_{k}, 1 \leq k \leq g$, such that $J=\bigcup_{k=1}^{g}\{k\} \times\left\{n_{k}+1, \ldots, m_{k}\right\}$. We will use the notation of Remark 2.4 .

Set $m=\sum_{k=1}^{g} m_{k}, n=m-\# J$. Assume that there are positive rational numbers $\alpha_{k}, \beta_{k}, 1 \leq k \leq g$, such that $\alpha_{k i}=\alpha_{k}$ if $1 \leq i \leq n_{k}, \alpha_{k i}=\beta_{k}$ if $n_{k}+1 \leq i \leq m_{k}$, and $\alpha_{k}>\beta_{k}, 1 \leq k \leq g$. Since $v\left(\theta_{k i}\right)=v\left(M_{k}\right)-v\left(x_{k i}\right)=v\left(M_{k}\right)-\alpha_{k i}$,

$$
\lim _{t \rightarrow 0} \vartheta(t) \in \Lambda_{J} .
$$


Let $\psi:(\mathbb{C} \backslash\{0\})^{n} \rightarrow \Lambda_{J}$ be the map defined by

$$
\psi\left(\varepsilon_{i j}\right)=\lim _{t \rightarrow 0} \vartheta(t) .
$$

The map $\psi$ has components $\psi_{k i}, 1 \leq i \leq n_{k}, 1 \leq k \leq g$. In order to prove the theorem it is enough to show that we can choose the rational numbers $\alpha_{k}, \beta_{k}$ in such a way that the Jacobian of $\psi$ does not vanish identically. We will proceed by induction in $k$. Let $k=1$. Since $\sum_{i=1}^{m_{1}} a_{11 i}<1, n_{1}=m_{1}$. Choose positive rationals $\alpha_{1}, \beta_{1}, \alpha_{1}>\beta_{1}$. There is a rational number $v_{0}<0$ such that $v\left(\theta_{1 i}\right)=v_{0}$, for all $1 \leq i \leq n_{1}$.

Assume that there are $\alpha_{k}, \beta_{k}$ such that $v\left(\theta_{k i}\right)=v_{0}$ for $1 \leq i \leq n_{k}$ and $v\left(\theta_{k i}\right)>v_{0}$ for $n_{k}+1 \leq i \leq m_{k}, k=1, \ldots, u$. Set

$$
\underline{\alpha}_{u+1}=\frac{\alpha_{u}+\sum_{k=1}^{u} \sum_{i=1}^{m_{k}}\left(a_{u+1, k, i}-a_{u k i}\right) \alpha_{k i}}{1-\sum_{i=1}^{n_{u+1}} a_{u+1, u+1, i}} .
$$

Since the special monomials are ordered by valuation and, by construction of $\Lambda_{J}$, $\sum_{i=1}^{n_{k}} a_{k k i}<1$ for all $1 \leq k \leq g, \underline{\alpha}_{u+1}$ is a positive rational number. Choose a rational number $\beta_{u+1}$ such that $0<\beta_{u+1}<\underline{\alpha}_{u+1}$. Set

$$
\alpha_{u+1}=\underline{\alpha}_{u+1}+\frac{\sum_{i=n_{u+1}+1}^{m_{u+1}} a_{u+1, u+1, i} \beta_{u+1}}{1-\sum_{i=1}^{n_{u+1}} a_{u+1, u+1, i}} .
$$

Then, $v\left(\theta_{u+1, i}\right)=v\left(M_{u+1}\right)-\alpha_{u+1}=v\left(M_{u}\right)-\alpha_{u}=v_{0}$ for $1 \leq i \leq n_{u+1}$.

Set $\widehat{M}_{k}=\prod_{i=1}^{k} \prod_{j=1}^{m_{k}} \varepsilon_{i j}^{a_{k i j}}, 1 \leq i \leq n_{k}, 1 \leq k \leq g$. With these choices of $\alpha_{k i}$, we have that

$$
\psi_{k i}=\frac{1}{\varepsilon_{k i}} \sum_{l=k}^{g} a_{k l i} \widehat{M}_{l}, 1 \leq i \leq m_{k}, 1 \leq k \leq g .
$$

Let $D$ be the Jacobian matrix of $\psi$. The matrix $D$ has $n_{r} \times n_{s}$ blocks $D_{r s}$, $1 \leq r, s \leq g$. If $r<s$, the entries of $D_{r s}$ are

$$
\frac{1}{\varepsilon_{r i} \varepsilon_{s j}} \sum_{l=s}^{g} a_{r l i} a_{s l j} \widehat{M}_{l}
$$

Moreover, $D_{r r}$ has entries

$$
\frac{\widehat{M}_{r}}{\varepsilon_{r i} \varepsilon_{r j}}\left(a_{r r i}\left(a_{r r j}-\delta_{i j}\right)+\sum_{l=r+1}^{g} a_{r r i} a_{r r j} \widehat{M}_{l}\right) .
$$

Let $m$ be the maximal ideal of the $\operatorname{ring} \mathcal{O}\left(\widehat{M}_{g}\right)$. If $r \leq s$ the entry $(i, j)$ of $D_{r s}$ belongs to the ideal generated by $\widehat{M}_{s} /\left(\varepsilon_{r i} \varepsilon_{r j}\right)$. Hence $\operatorname{det}\left(D_{r r}\right)$ belongs to the ideal $I_{r}$ generated by

$$
\left(\widehat{M}_{r}^{m_{r}} / \prod_{i=1}^{m_{r}} \varepsilon_{r i}\right)^{2}, 1 \leq r \leq g .
$$

Moreover, $\operatorname{det}(D)$ belongs to the ideal $I$ generated by

$$
\left(\prod_{l=1}^{g} \widehat{M}_{l}^{m_{l}} / \prod_{l=1}^{g} \prod_{i=1}^{m_{l}} \varepsilon_{l i}\right)^{2} .
$$

Let $\sigma$ be a permutation of $\left\{(1,1), \ldots,\left(1, m_{1}\right), \ldots,(g, 1), \ldots,\left(g, m_{g}\right)\right\}$. If there are $(r, i),(s, j)$ such that $\sigma(r, i)=(s, j)$ and $r \neq s$, the product of the entries $(r, i), \sigma(r, i)$ of $D$ belongs to the ideal $I m$. Therefore $\operatorname{det}(D)$ is congruent modulo $I m$ to the 
product of the determinants of the diagonal blocks $D_{r r}, 1 \leq r \leq g$. Moreover, $\operatorname{det}\left(D_{r r}\right)$ is congruent modulo $I_{r} m$ to the determinant of the matrix $D_{r}$ with entries

$$
\frac{\widehat{M}_{r}}{\varepsilon_{r i} \varepsilon_{r j}} a_{r r i}\left(a_{r r i}-\delta_{i j}\right) \text {. }
$$

By Lemma 2.8, $\operatorname{det}\left(D_{r}\right)$ equals the product of (2.27) by a nonvanishing complex number. Therefore there are $\lambda \in \mathbb{C} \backslash\{0\}$ and $\varepsilon \in m$ such that $\operatorname{det}(D)$ equals the product of (2.28) by a unit of $\mathcal{O}\left(\widehat{M}_{g}\right)$. Hence $\operatorname{det}(D)$ does not vanish identically and $\Lambda$ contains an open set of $\Lambda_{J}$. Since $\Lambda$ is a projective variety and $\Lambda_{J}$ is irreducible, $\Lambda$ contains $\Lambda_{J}$.

Theorem 2.10. Assume that $\sum_{i=1}^{m_{1}} a_{11 i}>1$. Set

$$
L=\bigcup_{k=1}^{g}\{k\} \times\left\{1, \ldots, m_{k}\right\}, \mathcal{I}=\bigcup_{k=1}^{g}\left\{\{k\} \times I: \sum_{j \in I} a_{k k j} \geq 1\right\} .
$$

The set $\Lambda$ is the union of the irreducible linear projective sets $\Lambda_{J}, J \in \mathcal{I}^{*}$, defined by the equations (2.24). The tangent cone of $Y$ equals $\{y=0\}$. The halo of $Y$ is the union of the cones $V_{J}, J \in \mathcal{I}^{*}$, where $V_{J}$ is defined by the equations $y=0$ and (2.25).

Proof. The proof is analogous to the proof of Theorem 2.9. On the first induction step we choose

$$
\beta_{1}=\left(\frac{1-\sum_{i=1}^{n_{1}} a_{11 i}}{\sum_{i=n_{1}+1}^{m_{1}} a_{11 i}}\right) \alpha_{1} .
$$

Hence $\beta_{1}<\alpha_{1}, v\left(\theta_{1 i}\right)=v(\eta)=0$ for $1 \leq i \leq n_{1}$ and $v\left(\theta_{1 i}\right)>0$ for $n_{1}+1 \leq i \leq m_{1}$. The rest of the proof proceeds as in the previous case.

Theorem 2.11. Assume that $\sum_{i=1}^{m_{1}} a_{11 i}=1$. Set

$$
L=\bigcup_{k=2}^{g}\{k\} \times\left\{1, \ldots, m_{k}\right\}, \mathcal{I}=\bigcup_{k=2}^{g}\left\{\{k\} \times I: \sum_{j \in I} a_{k k j} \geq 1\right\} .
$$

The set $\Lambda$ is the union of the irreducible projective algebraic sets $\Lambda_{J}, J \in \mathcal{I}^{*}$, where $\Lambda_{J}$ is defined by the equations (2.20) and (2.24).

There are integers $c, d_{i}$ such that $a_{11 i}=d_{i} / c, 1 \leq i \leq m_{1}$, and $c$ is the l.c.d. of $d_{1}, \ldots, d_{m_{1}}$. The tangent cone of $Y$ equals

$$
y^{c}-f(0)^{c} \prod_{i=1}^{m_{1}} x_{1 i}^{d_{i}}=0 .
$$

The halo of $Y$ is the union of the cones $V_{J}, J \in \mathcal{I}^{*}$, where $V_{J}$ is defined by the equations (2.25) and (2.29).

Proof. Following the arguments of Theorem 2.9 it is enough to show that $\Lambda_{J} \subset \Lambda$ for each $J \in \mathcal{I}^{*}$. Choose $J \in \mathcal{I}^{*}$. Let $\tilde{\Lambda}_{J}$ be the linear projective variety defined by the equations (2.24). We follow an argument analogous to the one used in Theorem 2.9. We have $n_{1}=m_{1}$. We choose positive rational numbers $\alpha_{1}, \beta_{1}$ such that $\beta_{1}<\alpha_{1}$. Then $v\left(\theta_{1 i}\right)=0$ for all $i=1, \ldots, m_{1}$. The remaining steps of the proof proceed as before. Hence

$$
\lim _{t \rightarrow 0} \vartheta(t) \in \tilde{\Lambda}_{J}
$$


Let $\psi:(\mathbb{C} \backslash\{0\})^{n} \rightarrow \tilde{\Lambda}_{J}$ be the map defined by (2.26). By Theorem 2.3 the image of $\psi$ is contained in $\Lambda_{J}$. By Lemma $2.8, \operatorname{det}\left(D_{1}\right)=0$. Let $D_{1}^{\prime}$ be the matrix obtained from $D_{1}$ by eliminating the $m_{1}$-th line and column. The argument of the proof of Theorem 2.9 works when we replace $D_{1}$ by $D_{1}^{\prime}$. Hence, $\Lambda_{J} \subset \Lambda$.

Let $Y$ be a quasi-ordinary hypersurface singularity.

Corollary 2.12. The set of limits of tangents of $Y$ depends only on the tangent cone of $Y$ and the topology of $Y$.

Corollary 2.13. If the tangent cone of $Y$ is a hyperplane, the set of limits of tangents of $Y$ depends only on the topology of $Y$.

Corollary 2.14. Let $x_{1}^{\alpha_{1}} \cdots x_{k}^{\alpha_{k}}$ be the first special monomial of $Y$. If $\alpha_{1}+\cdots+$ $\alpha_{k} \neq 1$, the set of limits of tangents of $Y$ depends only on the topology of $Y$.

Corollary 2.15. The triviality of the set of limits of tangents of $Y$ is a topological invariant of $Y$.

Proof. The set of limits of tangents of $Y$ is trivial if and only if all the exponents of all the special monomials of $Y$ are greater than or equal to 1.

\section{REFERENCES}

1. C. Ban, "Auréole of a quasi-ordinary singularity", Proc. Amer. Math. Soc., 120 (1994), 393-404. MR1186128 (94i:32055)

2. J. Lipman, "Quasi-ordinary singularities of embedded surfaces", Ph.D. thesis, Harvard University, 1965. MR2939573

3. J. Lipman, "Topological invariants of quasi-ordinary singularities", Mem. Amer. Math. Soc., No. 74, Amer. Math. Soc., Providence, RI, 1988, pp. 1-107. MR.954947 (89m:14001)

4. Lê Dũng Tráng and B. Teissier, "Limites d'espaces tangents en géométrie analytique", Comment. Math. Helv., 63 (1988), 540-578. MR966949 (89m:32025)

5. O. Neto, "A microlocal Riemann-Hilbert correspondence", Compositio Math., 127 (2001), No. 3, 229-241. MR1845036(2002k:32012)

6. M. Kashiwara and T. Kawai, "On holonomic systems of microdifferential equations. III. Systems with regular singularities", Publ. Res. Inst. Math. Sci., Vol. 17, No. 3 (1981), 813979. MR650216 (83e:58085)

DCet, Universidade Aberta, R. Escola Politecnica 141-147, 1269-001 Lisboa, Portugal - And - CMAF, Universidade de Lisboa, Av. Gama Pinto, 2, 1699-003 Lisboa, PORTUGAL

E-mail address: ant.arj@gmail.com

Departamento de Matemática and CMaf, Faculdade de Ciências da Universidade de Lisboa, Av. Gama Pinto, 2, 1699-003 Lisboa, Portugal

E-mail address: orlando60@gmail.com 